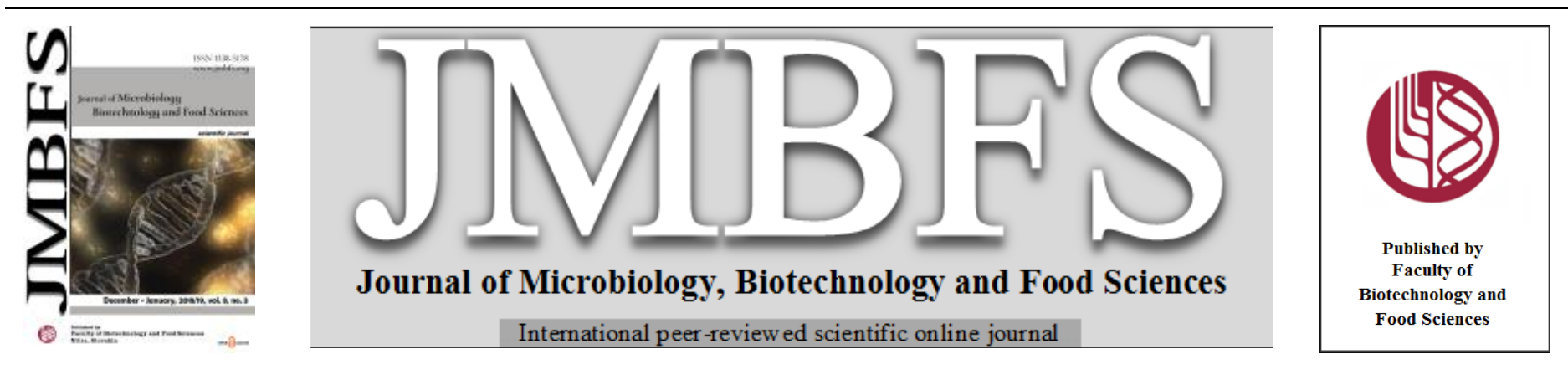

\title{
STANDARDISATION OF PAPER BASED PCR FOR DETECTION OF BACTERIA USING 16S rRNA GENE
}

\author{
Anushree Lokur*1, Surabhi Late ${ }^{2}$, Anjali Apte-Deshpande ${ }^{3}$
}

Address(es): Dr. Anushree Lokur,

${ }^{1}$ Ramnarain Ruia Autonomous College, Department of Microbiology, L. Nappo Road, Matunga, Dadar (East), Mumbai, Maharashtra - 400019, India. Phone: +91 9821331914.

${ }^{2,3}$ Central Dogma Pvt Ltd, 2, Richmond Park, Baner Road, Baner, Pune 411045.

*Corresponding author: aslokur@ruiacollege.edu

doi: 10.15414/jmbfs.2018-19.8.3.878-881

\section{ARTICLE INFO}

Received 10. 5. 2018

Revised 21.9. 2018

Accepted 24. 9. 2018

Published 1. 12. 2018

Regular article open $\partial_{\text {access }}$

\begin{abstract}
Reliable procedures are necessary to preserve, transport and test clinical, water or food samples, frequently requiring a challenging and costly cold chain to be in place. Effective disease surveillance or detection is thus severely hampered in resource-limited settings, such as those currently present in rural India, where procedures for appropriate laboratory based detection are suboptimal. Hence the recent scientific research is been focused to develop laboratory techniques/devices that can be applied in point of care settings. Point-of care (POC) techniques are getting increasingly popular, due to the advantages provided by them like ease of use, variety of applications, cost effectiveness and ease of disposal. This study deals with the standardization of method for microbial detection from sample collected on filter paper by direct amplification of a gene using PCR. Bacterial presence in a sample is confirmed by amplification of 16S rRNA gene as a proof of concept. 16S rRNA gene detection directly from sample spotted on Whatman filter paper no.3 without any pre-treatment to extract DNA is demonstrated, which is different from the currently practiced methods that include an additional step of DNA extraction from the paper. Robustness of the method was tested using Gram positive and Gram negative bacterial cultures and the sensitivity of detection on Whatman filter paper no. 3 was found to be $40-50$ cells.
\end{abstract}

Keywords: 16 S rRNA, PCR, Whatman paper, Point-of-care

\section{INTRODUCTION}

Point-of care (POC) techniques/devices are getting popularity worldwide because they can be effectively used in resource-limited settings. POC devices are used out of the laboratory settings and offer advantages like ease of use, variety of applications, cost effective and ease of disposal. The POC devices can be categorized as Instrumented and un-instrumented devices. Amongst uninstrumented devices, one of the commonly used methodology is filter paper.

Samples stored on the filter papers are typically used for identification of microbes or parasites. Most common practice is extraction of DNA from these samples followed by PCR to amplify specific gene which aids in identification. Although there are some reports where direct PCR is carried out without prior DNA extraction step. Certainly the biggest advantage of direct PCR is the fact that the extraction and purification of DNA from filter paper can be omitted, resulting in an overall saving of the time and chemicals required for DNA extraction step. Additionally, it can potentially avoid loss of DNA during extraction due to manual error or poor sample conditions which can intern affect the PCR reaction.

The aim of this study was to develop and optimize a direct paper based PCR technique for the detection of bacteria from water sample without the DNA extraction process. To establish the proof of concept for this technique, method development and optimization was done using 16S rRNA gene as a model.

$16 \mathrm{~S}$ rRNA gene is the most common housekeeping genetic marker used to study bacterial phylogeny and taxonomy for a number of reasons. These include: (i) its presence in almost all bacteria, often existing as a multigene family, or operons; (ii) the function of the 16S rRNA gene over time has not changed, suggesting that random sequence changes are a more accurate measure of time (evolution); and (iii) the $16 \mathrm{~S}$ rRNA gene (1500 bp) is large enough for informatics purposes. This gene consists of two regions - conserved and variable. The conserved regions are targeted by universal primers for the detection of the presence of microorganism in a given sample whereas the variable regions are targeted for identification of genus or species using specific primers (Hassan, El Enany, \& Rizk, 2014)

The current work is a proof of concept to establish direct amplification of $16 \mathrm{~S}$ rRNA gene from a sample applied on a Whatman paper to detect presence of microbes without extraction of genomic DNA. Universal primers were used to amplify the gene. The technique can be standardized for detection of particular organism using species specific /unique primers.

\section{MATERIAL AND METHODS}

\section{Cultures Used}

Genetically modified $E$. coli strains BL21 (DE3) and DH5 $\alpha$ were procured from Invitrogen (USA). Other bacterial cultures Streptococcus pyogenes, Staphylococcus aureus, Klebsiella pneumoniae, Citrobacter and Bacillus sp were laboratory maintained strains.

\section{Media and Reagents}

PCR reaction components, Taq polymerase and buffer were from Invitrogen, dNTPs were from Invitrogen. Nitrocellulose membrane was procured from Invitrogen, USA and Whatman filter paper no. 3 was from GE Healthcare Life Sciences, Luria Bertani (LB) agar components were procured from Himedia.

\section{Optimization of parameters for 16S rRNA gene PCR for detection of bacteria}

For optimizing the conditions for 16S rRNA gene PCR, it was important to evaluate the various components used in PCR reaction. Genetically modified $E$ coli strains (DH5 $\alpha$ and BL21 DE3) were used as a source of template DNA. Following sections describe the details about optimization of each component

\section{Primer Selection}

Genomic DNA (gDNA) of $E$. coli strains DH5 $\alpha$ and BL21 (DE3) were isolated using QIAamp DNA Mini Kit from Qiagen following the protocol recommended by the manufacturer. Additionally, isolated colony of both the strains of $E$. coli mentioned above, was picked up from LB agar plates and was re-suspended in $25 \mu \mathrm{L}$ of water in 2 distinct tubes. These tubes were kept in boiling water bath to lyse the cells. The boiled cell suspension was spun down and supernatant containing the gDNA was used as template. The gDNA extracted using both the 
above methods was used as template in the PCR. Two sets of universal primers with following sequences were synthesized (Sigma) for amplification of $16 \mathrm{~S}$ rRNA gene.

Set 1: F1: 5'ACT CCT ACG GGA GGC AGC AGT 3', R1: 5'TCA CCG GCC GTG TGT ACA AG-3' (Matsuda et al., 2011)

Set 2: F2: 5'GTG TAG CGG TGA AAT GCG 3', R2: 5'ACG GGC GGT GTG TAC AA3' (Sauer, Gallo, Kesselova, Kolar, \& Koukalova, 2005)

A typical PCR reaction was set up either using F1/R1 or F2/R2 primers, gDNA of DH5 $\alpha$ and BL21 (DE3) (from colony or extraction) along with optima concentrations of dNTP, Taq polymerase and buffer. PCR cycle program was set as $94^{\circ} \mathrm{C}$ for 5 minutes for initial denaturation followed by 35 cycles of $94^{\circ} \mathrm{C}$ for 30 seconds, $55^{\circ} \mathrm{C}$ for 30 seconds, $72^{\circ} \mathrm{C}$ for 30 seconds and final extension of $72^{\circ} \mathrm{C}$ for 7 minutes. Expected amplicon size for F1/R1 and F2/R2 primers was 1086 bp and 709bp respectively. Post amplification the amplified product was loaded on $1 \%$ agarose gel having ethidium bromide and the amplicon was visualized on UV transilluminator.

\section{Cell Lysis Optimization for Release of gDNA during PCR Cycle}

The major goal of the study was to establish a robust paper based PCR method without prior treatment of the sample.

Three isolated colonies of $E$. coli $\mathrm{DH} 5 \alpha$ were picked up from LB agar plates and were re-suspended in sterile water. $5 \mu \mathrm{L}$ of this suspension was directly spotted on the small strip of pre-sterilized Whatman filter paper no. 3 in a laminar airflow unit (LAFU) without prior boiling. The paper was air dried in LAFU to avoid any contamination and was directly suspended in the master mix containing appropriate concentration of buffer, dNTP, F2/R2 primers and Taq polymerase. Along with this reaction, a standard colony PCR of DH5 $\alpha$ culture was set up using a colony suspended in water and the mixture was boiled and post cell lysis, suspension was used as template in PCR master mix. $5 \mu \mathrm{L}$ of lysed cell suspension post boiling was then spotted on a pre-sterilized Whatman filter paper no. 3 as mentioned above and was allowed to air dry. This paper was also placed in a separate master mix and all the 3 reactions were processed for amplification. PCR cycling conditions and post amplification analysis was carried out.

\section{Bacterial Culture Volume Optimization for Paper Based PCR}

As the paper based PCR method for amplification of 16S rRNA gene was established using Whatman filter paper no. 3, it was important to optimize the lowest volume of culture that is sufficient to amplify $16 \mathrm{~S}$ rRNA gene. Since the paper was directly dipped in $25 \mu \mathrm{L}$ of PCR master mix in a PCR tube, there was limitation of the size of the paper that can be used for the reaction which further limits the volume of culture that can be accommodated on that small piece of paper. Earlier experiments had shown that $5 \mu \mathrm{L}$ culture could be used to get desired amplicon; the volumes were reduced below $5 \mu \mathrm{L}$. DH5 $\alpha$ colony suspension was prepared in sterile water. This suspension, without boiling was directly spotted on 3 independent presterilized small strips of Whatman filter paper no. 3 as 1,3 and $5 \mu \mathrm{L}$. The strips were allowed to air dry in LAFU and were directly dipped in $25 \mu \mathrm{L}$ of PCR master mix and the amplification and detection was carried out. Standard colony PCR of the same culture was also carried out along with this reaction as a positive control.

\section{Paper Based PCR Method Validation}

Determination of Limit of detection (LOD) using $E$. coli DH5a and BL21 (DE3) as model cultures

DH5 $\alpha$ suspension of 0.1 abs was prepared from overnight culture. It was diluted serially ten-fold up to $10^{-6}$ and $5 \mu \mathrm{L}$ of each suspension was spotted on presterilized independent Whatman filter paper no.3 strips and the strips were air dried in LAFU. These strips were dipped in master mix having all reaction components and F2/R2 primers and PCR amplification was carried out, except the cycles were increased to 40 from 35 cycles and reaction volume was shifted to $30 \mu \mathrm{L}$. Amplicon was loaded on agarose gel and was detected on UV transilluminator.

Viable count of all the dilutions was done by Miles and Misra's technique.

\section{Optimal annealing temperature determination}

To evaluate the optimal annealing temperature for primer set F2/R2, a gradient PCR program was set up in thermal cycler with annealing temperature range of $55-58^{\circ} \mathrm{C}$ where as other cycling conditions are maintained.

As a model organism, single colony of $E$. coli strain BL21 (DE3) was suspended in $15 \mu \mathrm{L}$ of sterile water and was further diluted 10 fold serially up to $10^{-6}$. Al these dilutions were spotted on presterilized Whatman filter paper no. 3 and processed as mentioned in LOD section. All these dilutions were exposed to 55 ${ }^{\circ} \mathrm{C}$ annealing temperature whereas the 6th dilution was also used at 56, 57 and $58^{\circ} \mathrm{C}$ annealing temperatures. The amplification was visualized.

\section{Robustness}

$16 \mathrm{~S}$ rRNA gene is a house keeping gene for all the bacteria, whether Gram positive or Gram negative. To ensure the specificity of the paper based PCR various Gram positive and Gram negative bacterial colony suspensions were prepared and were spotted on pre-sterilized Whatman filter paper no.3 as mentioned earlier. The bacteria used for this study were Streptococcus pyogenes, Klebsiella pneumoniae, Citrobacter and Bacillus sp. Each of these cultures were streaked on sterile Luria agar plate to achieve single isolated colony. Single colony of each of these cultures was picked up and suspended in $15 \mu \mathrm{L}$ of sterile water and it was further serially diluted to get $40-50$ cells $/ 5 \mu \mathrm{L}$ for each of the five cultures. $5 \mu \mathrm{L}$ of each suspension was spotted on pre-sterilized independent Whatman filter paper no.3 strips and the strips were air dried in LAFU. These strips were dipped in master mix and PCR was carried out as mentioned earlier in case of $E$. coli DH5 $\alpha$. The experiment was carried out with 2 different master mixes, one with F1/R1 primer set and another with F2/R2 primer set. An undiluted colony suspension of each culture served as a positive control along with paper based PCR with 40-50 cells.

\section{RESULTS AND DISCUSSION}

Optimization of parameters for 16S rRNA gene PCR for detection of bacteria

\section{Primer Selection}

A pair of universal primers was selected from the highly conserved region of the $16 \mathrm{~S}$ rRNA sequence. The up-stream primer corresponded to $\mathrm{C} 1$ region nucleotides 358 to 378 and the downstream primer corresponded to $\mathrm{C} 6$ region nucleotides 1444 to 1425 (Labeled as F1/R1 for this study). The primers were used to amplify the region which gave the amplicon of size $1086 \mathrm{bp}$. Both genetically modified E. coli DH5 $\alpha$ and BL21 (DE3) strains could give desired amplicon irrespective of whether genomic DNA was extracted or a colony was used as a source of DNA (Fig 1). Earlier, these primers were also used by Matsuda et al. (Matsuda et al., 2011) for suspected blood infections and by Liu et al. (Liu et al., 2005) for the bacterial infection in CSF. The aim here was to establish the primer pair which is suitable for amplification of 16S rRNA gene which can be used in paper based PCR method that was being developed for detection of microbes. Also another set of universal primer was evaluated for their efficiency to amplify the bacterial DNA. 16S rRNA primers used by Sauer et al. (Sauer, Gallo, Kesselova, Kolar, \& Koukalova, 2005) for detection of pathogenic bacteria causing prosthetic joint infections were synthesized. The authors have claimed that the primer pair is highly specific for broad range of bacterial pathogens and can be used with variety of clinical specimens. Since the ultimate goal of the current study was to establish a robust paper based PCR method to detect microbes from variety of food and clinical samples, it was essential to demonstrate the primer pair that can be used commonly with broad range of samples. In the current study this primer pair was labeled as F2/R2.

When F2/R2 was used in the PCR for amplification of 16S rRNA gene from genetically modified $E$. coli strains, amplicon of 709 bp was detectable on the agarose gel electrophoresisn (Fig1). Since both the primer pairs were highly specific for $16 \mathrm{~S}$ rRNA gene, one primer pair (F2/R2) was used in subsequent experimentation.
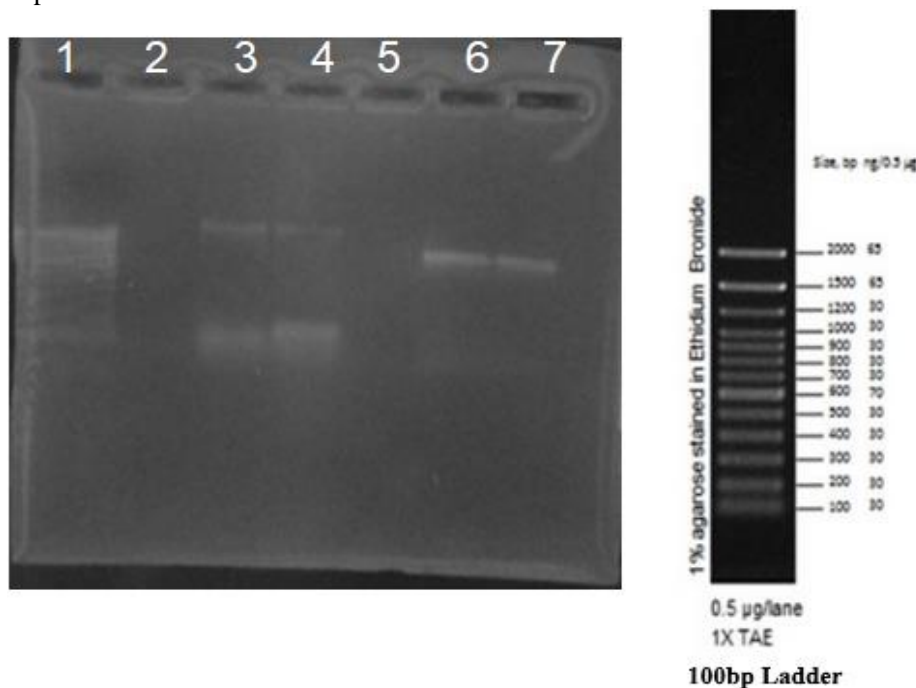

Figure 1 Amplification of 16sRNA gene of E. coli strain DH5 $\alpha$ and BL21DE3 using universal primers

Lane 1: 100 bp ladder, Lane 2: Negative control for F1/R1, Lane 3: 16srNA gene of DH5a amplified with F1/R1, Lane 4: 16srRNA gene of BL21DE3 amplified with F1/R1, Lane 5: Negative control for F2R2, Lane 6: 16srNA gene of DH5 $\alpha$ amplified with F2/R2, Lane 7: 16srRNA gene of BL21DE3 amplified with F2/R2 


\section{Optimization of Cell Lysis for PCR}

In a standard colony PCR reaction the colony suspension is boiled to lyse the cells and release the genomic DNA which is subsequently amplified with gene specific primers. To optimize this cell treatment for paper based PCR reaction, the colony suspension was boiled first and then spotted on pre-sterilized Whatman filter paper no.3. To evaluate the possibility of eliminating this lysis step, a reaction was also set up where the colony suspension was not boiled and directly spotted onto pre-sterilized Whatman filter paper no.3. All the three conditions could give the amplification of 16S rRNA (Data not shown) which implies that the boiling treatment for cell lysis and release of genomic DNA can be eliminated. This may be because the first denaturation step in PCR cycling condition helps in cell lysis and release of genomic DNA which is subsequently amplified. Hence the boiling of cell culture was not carried out in further experiments and the cultures were directly spotted on pre-sterilized Whatman filter paper no. 3 for paper based PCR.

\section{Culture volume Optimisation}

Paper-based PCR for amplification of 16S rRNA was optimized with Whatman filter paper no.3 and without prior cell treatment. Since the final PCR reaction is carried out in small volumes $(30 \mu \mathrm{L})$, it was essential to reduce the size of the Whatman filter paper no. 3 which is being dipped in the PCR tube and stays in the tube throughout the amplification. It was hence decided to load different volumes of cultures on the paper to evaluate the lowest possible volume of culture that can give the positive reaction. The amplification of $16 \mathrm{Sr}$ RNA gene could be achieved with as low volume as $1 \mu \mathrm{L}$ of culture on paper, using the PCR conditions optimized so far. Standard colony PCR reaction without use of Whatman filter paper no. 3 served as a positive control.

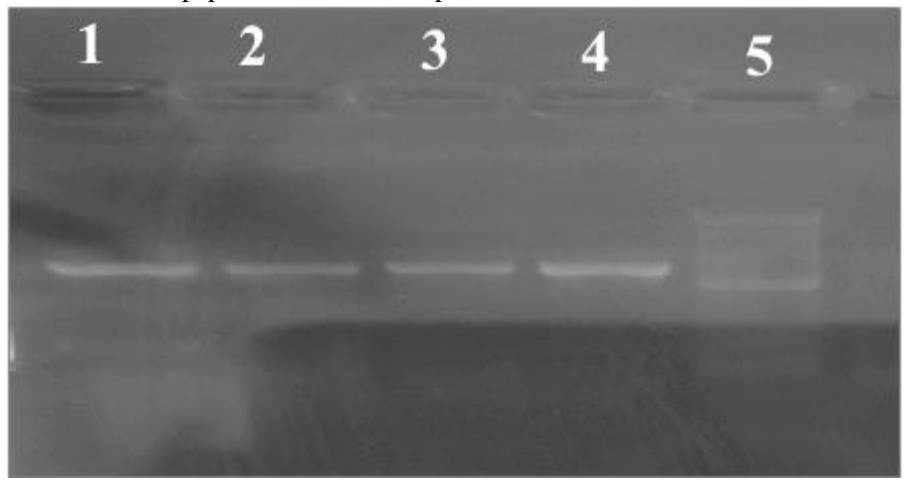

Figure 2 E. coli $\mathrm{DH} 5 \alpha$ culture volume optimization for amplification of 16srRNA gene

Lane 1: DH5 $\alpha$ - colony PCR, Lane 2: Amplicon when $5 \mu$ l culture spotted, Lane 3: Amplicon when $3 \mu \mathrm{l}$ culture spotted, Lane 4: Amplicon when $1 \mu \mathrm{l}$ culture spotted on presterilized Whatman paper No 3, Lane 5: 100 bp ladder

\section{Determination of limit of detection (LOD) for 16S rRNA gene in $E$. col strains DH5 $\alpha$ and BL21 (DE3)}

A single colony of $E$. coli strains DH5 $\alpha$ contains $\sim 9 \times 10^{8}$ cells $\mathrm{mL}^{-1}$ which was serially diluted up to $10^{-6}$ to give $\sim 900$ cellsmL ${ }^{-1}$. Since for amplification reaction, $5 \mu \mathrm{L}$ of this suspension was spotted on pre-sterilized Whatman filter paper no.3 and 16S rRNA amplification could be achieved, that indicates the LOD to be 5 cells for $E$. coli strains DH5 $\alpha$. Since the intensity of amplicon band is very low in case of $10^{-6}$, ideally a previous dilution of $10^{-5}$ can be safely detected which is approximately 50 cells. Similarly for BL21 (DE3), a single colony suspension contains $\sim 4.5 \times 10^{9}$ cells $\mathrm{mL}^{-1}$ which was serially diluted tenfold up to $10^{-6}$ to give $\sim 4500$ cells $\mathrm{mL}^{-1}$. Since $5 \mu \mathrm{L}$ of this suspension on paper could amplify the gene, the LOD for BL21 (DE3) was also same as that of DH50 (Data not shown but dilution scheme was followed for detection of other organisms as mentioned in robustness section).

\section{Annealing temperature optimization for $16 \mathrm{~S}$ rRNA amplification from $E$. coli DH5a culture}

Earlier data has shown weak amplification when the E. coli DH5 $\alpha$ culture was serially diluted to $10^{-6}$ and spotted on paper. It was hence necessary to optimize the primer annealing temperature to get clearly detectable amplicon on agarose gel. The recommended annealing temperature of F2/R2 universal primers was $55^{\circ} \mathrm{C}$. To optimize this annealing temperature for better amplification, a gradien PCR was set up with the range $56-58^{\circ} \mathrm{C}$ with only $10^{-6}$ dilution spotted on the paper which served as a template DNA. Good intensity amplicon was visible in PCR with annealing temperature of $57^{\circ} \mathrm{C}$ (Data not shown). So here after, $57^{\circ} \mathrm{C}$ was used as the optimal annealing temperature.
16S rRNA gene amplification in Gram positive and Gram negative bacteria using paper based PC (Robustness)

To ensure the robustness of the developed paper based PCR method, Gram positive and Gram negative single colony bacterial suspensions were prepare and were diluted up to $10^{-5}$ dilutions which corresponds to $\sim 50$ cells. These were spotted on the pre-sterilized paper and were amplified using both sets of primers (F1/R1 and F2/R2).

For every culture, all dilutions and undiluted colony suspensions were independently amplified using F2/R2 primer pair. It is clearly seen in (Fig 3a) that $16 \mathrm{~S}$ rRNA gene can be amplified even post dilution of both Gram positive and negative bacterial culture up to 50 cells. Although, in some cases of diluted cultures, the intensity was relatively low; this could be due to the cell number being just around or below the detection limit. The robustness of the method was also tested using different set of primers (F1/R1) with undiluted cultures of gram positive and negative bacteria spotted on paper. All the cultures could give the amplicon of $\sim 1 \mathrm{~Kb}$ which is the expected size with F1/R1 primers (Fig 3b).

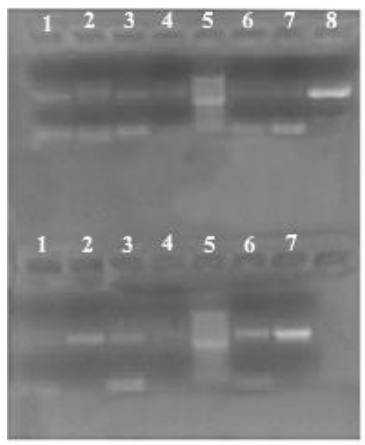

(a)

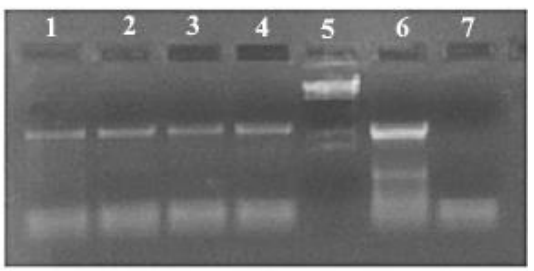

(b)
Figure 3 16S rRNA gene amplification in Gram positive and Gram negative bacteria using paper based PCR

(a) Amplification with F2/R2 primer pair Top half: For all the cultures positive control is single colony suspension and test is 40-50 cells spotted on Whatman filter paper no.3. (Left to Right) Lane $1 \& 2$ : K. pneumoniae positive control and Test, Lane 3 \& 4: Citrobacter positive control and Test, Lane 5: 100 bp ladder, Lane 6: negative control with Whatman, Lane 7: negative control water, Lane 8: positive control (DH5 $\alpha$ genomic DNA PCR); Bottom half: (Left to Right) Lane $1 \&$ 2: S. pyogenes Test and positive control, Lane 3 \& 4: Bacillus positive control and test, Lane 5: 100 bp ladder, Lane 6 \& 7: E. coli DH5 $\alpha$ Test and positive control; (b) Amplification with F1/R1 primer pair (Left to Right) Lane 1: S.pyogenes, Lane 2: Bacillus, Lane 3: Citrobacter, Lane 4: K. pneumoniae Lane 5:100 bp ladder, Lane 6: DH5 $\alpha$ genomic DNA PCR (positive control), Lane 7: negative control

\section{CONCLUSION}

16S rRNA gene detection directly using Whatman filter paper no. 3 without any pre-treatment to extract DNA from paper is demonstrated above as a proof of concept. The method is robust, sensitive and can detect up to 40-50 cells on the paper. The primers used for the amplification were universal primers and can be used for detection of presence or absence of bacteria in any sample. This technique can detect bacterial contamination in samples which are meant to be sterile like injectables, oral syrups, tissue culture media etc. The technique can be standardised for detection of particular species by using species specific variable regions in the gene.

This method can be used for analysis of samples brought from point of care in remote locations. The processing can be done in the central laboratories in tier 2 or tier 3 cities which have basic laboratory facilities such as basic PCR machine which is not very costly. The procedure does not need highly skilled workers for conducting the test. Additionally, specialized filter paper which is normally used for collection of samples like Whatman 903 is also replaced in this method by a normal Whatman filter paper no. 3 which is easily available at a lower cost. The detection of microbes can be done in a short span as compared to traditional microbiological methods. Therefore this direct paper based PCR method is versatile and can be used for applications in resource limited settings to applications in fully equipped central laboratories in developed countries.

Acknowledgments: We thank Ramnarain Ruia Autonomous College for their support and for providing us with the facilities.

\section{REFERENCES}

BU, Y., HUANG, H., \& ZHOU, G. (2008). Direct polymerase chain reaction (PCR) from human whole blood and filter-paper-dried blood by using a PCR buffer with a higher pH. Analytical Biochemistry, 375(2), 370-372 https://doi.org/10.1016/j.ab.2008.01.010 
HASSAN, R., EL ENANY, M., \& RIZK, H. (2014). Evaluation of broad-range $16 \mathrm{~S}$ rRNA PCR for the diagnosis of bloodstream infections: two years of experience. The Journal Of Infection In Developing Countries, 8(10) 1252-1258. https://doi.org/10.3855/jidc.4867

LIU, Z., LUO, J., BAI, Q., MA, M., GUAN, G., \& YIN, H. (2005). Amplification of $16 \mathrm{~S}$ rRNA genes of Anaplasma species in China for phylogenetic analysis. Veterinary Microbiology, 107(1-2), 145-148 https://doi.org/10.1016/j.vetmic.2005.01.007

MATSUDA, K., IWAKI, K., GARCIA-GOMEZ, J., HOFFMAN, J., INDERLIED, C., MASON, W., \& IWAKI, Y. (2011). Bacterial Identification by 16S rRNA Gene PCR Hybridization as a Supplement to Negative Culture Results. Journal Of Clinical Microbiology, 49(5), 2031-2034. https://doi.org/10.1128/JCM.00615-10

MCCABE, E. (1991). Utility of PCR for DNA analysis from dried blood spots on filter paper blotters. Genome Research, 1(2), 99-106.

http://dx.doi.org/10.1101/gr.1.2.99

MOLINA, F., LÓPEZ-ACEDO, E., TABLA, R., ROA, I., GÓMEZ, A., \&

REBOLLO, J. (2015). Improved detection of Escherichia coli and coliform bacteria by multiplex PCR. BMC Biotechnology, 15(1), 48 http://dx.doi.org/10.1186/s12896-015-0168-2

POLSKI, J., KIMZEY, S., PERCIVAL, R., \& GROSSO, L. (1998). Rapid and effective processing of blood specimens for diagnostic PCR using filter paper and Chelex-100. Molecular Pathology, 51(4), 215-217. http://dx.doi.org/10.1136/mp.51.4.215

SAUER, P., GALLO, J., KESSELOVA, M., KOLAR, M., \& KOUKALOVA, D. (2005). Universal primers for detection of common bacterial pathogens causing prosthetic joint infection. Biomedical Papers, 149(2), 285-288. http://dx.doi.org/10.5507/bp.2005.043

SMIT, P., ELliOTT, I., PEELING, R., MABEY, D., \& NEWTON, P. (2013) An Overview of the Clinical Use of Filter Paper in the Diagnosis of Tropical Diseases. American Journal Of Tropical Medicine And Hygiene, 90(2), 195-210. http://dx.doi.org/10.4269/ajtmh.13-0463 